\title{
Endoscopic management of intraventricular shunt-related cystic compartment in paediatric patients
}

\section{Ahmed Zaher, Amr Farid Khalil, Mohamed State, Hatem Badr}

\author{
* Department of Neurosurgery, Faculty of Medicine, Mansoura \\ University, EGYPT
}

\begin{abstract}
Background. Intraventricular uniloculated compartments are relatively seen frequently in children previously treated with ventriculoperitoneal shunts for hydrocephalus. These compartments may present before shunt insertion and even as an end result to the shunt gliosis. Although the availability of different surgical modalities for the management of such loculations but till now there is no consensus about a single therapeutic approach for its management. Objective: The aim of this study is to assess the role of neuroendoscopy in management of uniloculated compartments, in pediatric cases who underwent shunting for hydrocephalus, to avoid multiple shunt insertion and even to eliminate the need for the preexisting shunt system.
\end{abstract}

Patients and Methods. We retrospectively studied 44 consecutive previously shunted patients with isolated intraventricular compartment who underwent 58 endoscopic procedures by a single surgeon during the period between February 2015 and February 2018. These procedures included endoscopic cyst fenestration, intraventricular septum pellucidotomy, foraminoplasty and additionally third ventriculostomy in some cases was added to the previous procedures. Multiloculated hydrocephalus and cysts related to the tumours were excluded. Clinical, radiological and surgical data were assessed.

Results. The most common cause of a single loculation in those patients was neuroepithelial cyst ( 20 cases) followed by post shunt isolated compartment (12 cases), meningitis ( 8 cases) and four cases of intraventricular haemorrhage. The patient's age varied from two months up to seven years (mean $=3.2$ years). The follow-up period was ranged from (2-49 months) with the mean of 12.3 months. The overall success of restoration of communication between the uniloculated compartment and the ventricular system was achieved in 36 patients (81.8\%), with $28(63.6 \%)$ patient needed only one shunt and eight (18.2\%) patients became independent of their previous shunts. Fourteen repeated endoscopic procedures were considered necessary in ten patients for either reopening of a closed stoma and/or shunt revision and finally, eight patient (18\%) required additional shunt insertion during follow up period. The complications encountered were minor and reversible (CSF leak in two cases, infection in two cases, subdural fluid collection in four cases) with no mortality.

Conclusion. Minimal invasive endoscopic technique is a useful operative alternative to control uniloculated compartments in patients with previous ventriculoperitoneal shunts. It does not only simplify the existing shunt system by restoration of the communication between the loculated compartment and the draining shunt but it may eliminate the need for external shunt in some cases as well.

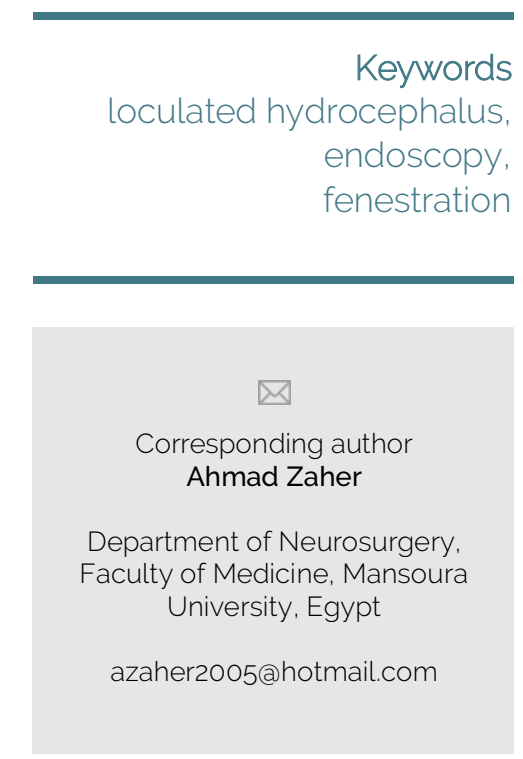

Copyright and usage. This is an Open Access article, distributed under the terms of the Creative Commons Attribution Non-Commercial No Derivatives License (https://creativecommons org/licenses/by-nc-nd/4.0/) which permits noncommercial re-use, distribution, and reproduction in any medium, provided the original work is unaltered and is properly cited.

The written permission of the Romanian Society of Neurosurgery must be obtained for commercial re-use or in order to create a derivative work.

ISSN online 2344-4959

(C) Romanian Society of Neurosurgery

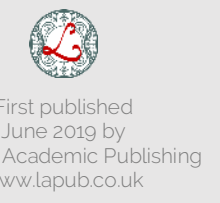




\section{INTRODUCTION}

Intraventricular septation may lead to formation of an isolated CSF cystic component which may become enlarged even if the shunt system is functioning. Such enlarging intraventricular loculation may be attributed to altered CSF dynamics as result of separation between the ventricular catheter tip and the site of CSF production [14].

Variable anatomical and radiological forms of loculations were described according to the site of obstruction and consequent CSF accumulation including either uniloculated or multiloculated. Identification and distinction between the two forms is crucial because everyone has a particular different pathogenesis, treatment and prognosis [7].

Loculated hydrocephalus usually develops after episodes of neonatal meningitis or germinal matrix hemorrhage. Occlusion of the foramen of Monro, the aqueduct, and the outlet foramina of the fourth ventricle may result from shunt infection and its consequences like ventriculitis and ventricular septations. However, isolated intraventricular compartment may evolve after insertion of ventriculoperitoneal shunt via other mechanisms rather than shunt infection $[11,14]$.

Direct ependymal trauma during shunt insertion, collapse of shunted ventricular compartment with subsequent obstruction of the catheter tip perforations by proliferating fibrillary astrocytes and over drainage of existing shunt may play important roles that may stimulate the enlargement of an isolated shunt related compartment [14].

The management of such complex condition is challenging to most neurosurgeons. The main goal of treatment is to elevate the manifestation of increased intracranial pressure by creating a free connection between the isolated compartment and the ventricular system cavity via converting them into a solitary component that will be drained by a single ventricular catheter with little morbidity and mortality $[8,19]$.

Multiple treatment strategies for intraventricular loculation are available but the best choice of the proper procedure remains controversial. This group of patients presented with intraventricular compartments related to ventriculoperitoneal shunts often require multiple and repeated operations. These patients usually necessitate either traditional shunt revisions alone or in combination with other procedures like insertion of multiple perforated catheter, craniotomy with transcallosal fenestration, stereotactic aspiration, endoscopic cyst fenestration, septostomy, foraminoplasty or third ventriculostomy $[18,20]$.

Recent advancement of neuroendoscopy and the higher incidence of complication associated with traditional shunt revision or open craniotomy have shifted many neurosurgeons to the different endoscopic techniques as simple and minimal invasive alternatives to solve this complex condition [9].

\section{PATIENTS AND METHODS}

From February 2015 to February 2018, forty-four consecutive paediatric cases presented with shunt related cystic compartments and a preexisting ventriculoperitoneal shunts were operated at "Neurosurgical Department of Mansoura University Hospitals" by a single surgeon using neuroendoscopic techniques. Our study included 28 boys and 16 girls; their ages ranged from two months to seven years (mean 3.2 years). The follow up period renged from (2-49 months) with the mean of 12.3 months. Cases of multiloculated hydrocephalus or uniloculated hydrocephalus without previous shunt insertion were excluded from our study. Tumour related cysts were also excluded.

We retrospectively reviewed the patient's data retrieved from the patient charts which included: patient history, clinical examination, radiological investigations, operative data, patient outcome, shunt independency, the need for additional shunts and the prerequisites to repeat endoscopic procedures.

Plain x-ray to the shunt system, CT scan and MRI brain with or without gadolinium were done to all patients preoperatively. CSF sample was obtained and examined to exclude CSF or shunt infection prior to endoscopic procedures.

Endoscopic surgery was indicated when the patients had clinical deterioration in the form of progressive enlargement of head size, development of manifestations of intracranial hypertension, worsening of the focal neurological deficits, gait disturbance or refractory epilepsy. The previous clinical manifestations had been correlated with radiological signs of the cyst enlargement and/or shunt malfunctioning. Cognitive dysfunction was not 
considered as an indication for surgical management.

Our aim of endoscopic surgery was to connect the isolated compartment to the ventricular cavity in order to drain both of them by the same shunt in order to simply the pre-existing shunt device and to avoid implanting another device whenever possible.

In all patients, surgery was done under general anaesthesia. The entry point was tailored individually in every case according to the cyst location and the location of the previous shunt entry point. A proper burr-hole location and the direct surgical corridor were the most critical points for proper cyst fenestration and shunt revision simultaneously.

Different angles rigid lens endoscopes $\left(0^{\circ}, 30^{\circ}\right.$, $70^{\circ}$ Karl-Storz rigid Gaab System) were used. The endoscopic sheath has an outer diameter of $6 \mathrm{~mm}$ and working channel of $2 \mathrm{~mm}$. After endoscopic introduction, continuous irrigation was essential for clear identification of key anatomical landmarks and proper visualization of cyst wall as well as the proximal end of the pre-existing ventricular catheter. Initial cyst fenestration was performed in a thin, translucent and relatively a vascular segment of the cyst wall by the use of bipolar electrocoagulation. Dilation and enlargement of the initial stoma was continued by with the aid of Fogarty balloon catheter, punches, and sharp scissor to fashion a wide stoma that secure a free CSF passage and minimize the incidence of reclosure of the stoma. Coagulation and devascularization of the cyst wall were done in order shrink the cyst wall and hold the cyst growth.

Other endoscopic procedures like endoscopic revision of the shunt, septum pellucidotomy, foramioplasty and third ventriculostomy were done in combination with endoscopic cyst fenestration to advance the communication of CSF isolated compartments.

Any bleeding point was secured with continuous irrigation, bipolar electrocoagulation and tamponading with embolectomy balloon catheter to achieve a non-vascular surgical field as well as the site of fenestration. At the end of the surgery, the endoscopic sheath was retrieved under continuous irrigation to clear out the surgical field from any blood clots or any surgical debris.

Clinical outcome was defined as improved, unchanged or worsened. Developmental delay was not considered in post-operative clinical outcome assessment. Epilepsy was defined to be improved if the attacks were controlled after withdrawal or even reduction of the dose of antiepileptic drugs. If the head circumference remained stable or slightly reduced in size, this was considered as a postoperative sign of improvement.

Routine postoperative CT brain was done to all patients twenty-four hours after surgery. Follow up $\mathrm{CT}$ and MRI brain were done routinely 3 months after surgery. The procedure was considered to be successful when endoscopic procedure was able to restore the continuity between the cyst and the ventricular system cavity without the need to insert additional shunt devices. Repeated endoscopic procedures were considered when the patient experienced recurrence or progression of his clinical manifestations that were concomitant with progressive cyst and/or ventricular enlargement on follow up CT and/or MRI images.

\section{RESULTS}

Progressive enlargement of the head size was the most common presenting symptom in our patients (28 cases, 63.6\%) followed by seizures (14 cases, $31.8 \%)$, headache (10 cases, 22.72\%), focal neurological deficits (10 cases, $22.72 \%$ ) and gait disturbance in 6 patients (13.6\%) (TABLE 1).

The non-colloidal neuroepithelial cysts were the most prominent underlying cause of intraventricular unilateral compartments with preexisting shunts in our study (Twenty cases, 45.5\%) while uniloculated cysts related to either previous shunt insertion or shunt infection represented the second most common cause (Twelve cases, 27.3\%) followed by meningitis (eight cases,18.2\%) and neonatal intraventricular haemorrhage (four cases, 9\%) (TABLE 2) .

Endoscopic fenestration of cyst wall was done in all patients. Other additional endoscopic procedures that were required to be done simultaneously with the endoscopic cyst fenestration included either endoscopic third ventriculostomy (six patients), septum pellucidotomy (ten patients) or foraminoplasty (six patients).

During the previous endoscopic procedures, shunt revisions were indicated in only six patients (33\%) who had non-colloidal neuroepithelial cysts whenever 20 out of 24 patients (83\%) of shunt related cysts, postmeningitic cysts and post- 
haemorrhagic cysts required endoscopic shunt revisions.

Throughout the follow up periods, the previous endoscopic procedures were successful to restore the connection between the isolated compartments and the ventricular system cavities in 36 patients (81.8\%).

Simplification of the previous shunt systems were achieved in 28 patients (63.6\%) who required only a single device, while eight patients needed additional shunt system, the remaining eight cases (18.2\%) became shunt independent after previous endoscopic procedures and shunt removal (TABLE 2).

Fourteen repeated endoscopic procedure were done in 10 patients for either reopening of the stoma in ten procedures or shunt revisions in the remaining other procedures. ( 2 cases due to gliosis and 2 due to depris). Finally, eight (18.2\%) patients required additional shunt insertion after failure of restoration of a proper connection between the isolated compartments and the ventricular system cavities with failure of a single shunt to drain the dilated cavities.
As regard clinical outcome, most patients $n=28$ (63.6\%) presented with progressive head enlargement showed either stabilization or slight reduction in their head size. Headache was improved in eight patients from 10 patients (80\%) with only two patients sustained to complain of headache after successful fenestration. Ten from 14 patients (72\%) presented with epilepsy achieved clinical improvement by either absence of their epilptogenic attacks after withdrawal of antiepileptic treatment or reduction of the doses of their treatment regimens, whenever two patients (14.2\%) remain dependent on the same drug regimen and another two patients required addition of another antiepileptic drug to control his seizures.

We had no mortalities among our patients whenever the procedure related morbidities were minimal including two of CSF leakage, two cases of superficial wound infection and four cases of subdural fluid collection.

TABLE 1. Clinical presentation and postoperative clinical outcome.

\begin{tabular}{|l|l|l|l|l|}
\hline \multicolumn{1}{|c|}{ Clinical presentation } & \multicolumn{1}{|c|}{ No. (\%) } & \multicolumn{3}{c|}{ Clinical outcome } \\
\hline Head enlargement & $28(63.6 \%)$ & $14(50 \%)$ & $12(43 \%)$ & $2(7 \%)$ \\
\hline Seizures & $14(31.8 \%)$ & $10(72 \%)$ & $2(14 \%)$ & $2(14 \%)$ \\
\hline Headache & $10(22.7 \%)$ & $8(80 \%)$ & $2(2 \%)$ & 0 \\
\hline Focal neurological deficit & $10(22.7 \%)$ & $6(60 \%)$ & $4(40 \%)$ & 0 \\
\hline Gait disturbance & $6(13.6 \%)$ & $4(66.7 \%)$ & $2(33.3 \%)$ & 0 \\
\hline
\end{tabular}

TABLE 2. Number of required shunts and repeated endoscopic procedures in relation to different etiologies.

\begin{tabular}{|l|c|c|c|c|c|}
\hline \multicolumn{1}{|c|}{ Etiology } & No. of & \multicolumn{3}{|c|}{ No. of required shunt } \\
\cline { 3 - 5 } & patients & No shunt & One shunt & Additional shunt & Repeated \\
endoscopic
\end{tabular}




\begin{tabular}{|l|c|c|c|c|c|}
\hline Intraventricular age & 4 & 0 & 2 & 2 & 4 \\
\hline Total no. and \% & $44(100 \%)$ & $8(18 \%)$ & $28(64 \%)$ & $8(18 \%)$ & $14(24 \%)$ \\
\hline
\end{tabular}

TABLE 3. Repeated endoscopic procedure in ten patients.

\begin{tabular}{|c|c|c|}
\hline Type of the procedure & No. & $\%$ \\
\hline Closed fenestration & 10 & 71.4 \\
\hline Shunt revision & 4 & 28.6 \\
\hline Gliosis & 2 & 14.3 \\
\hline Obstruction form debris & 2 & 14.3 \\
\hline
\end{tabular}

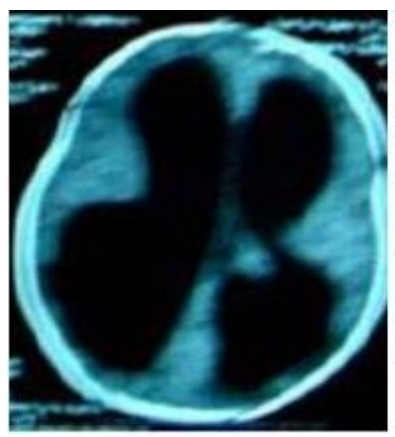

(a)

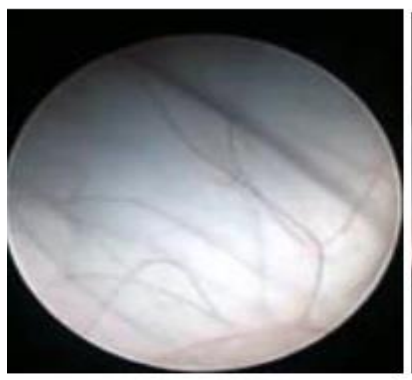

(e)

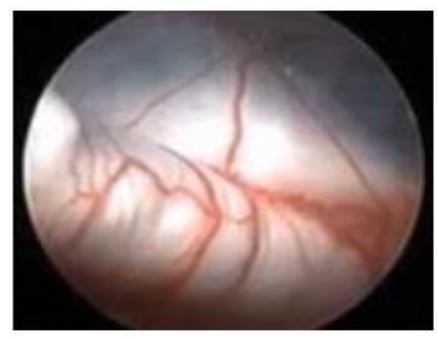

(i)

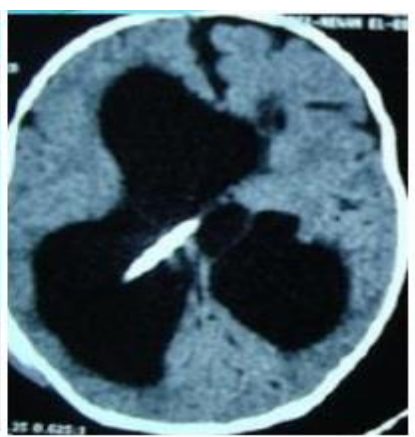

(b)

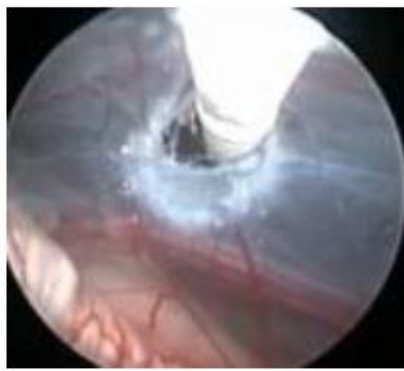

(f)

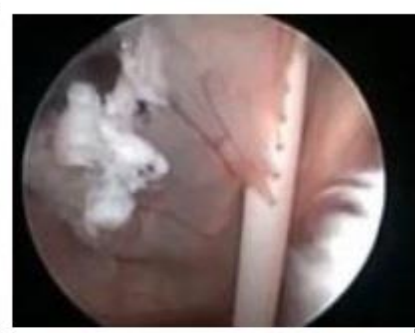

(j)

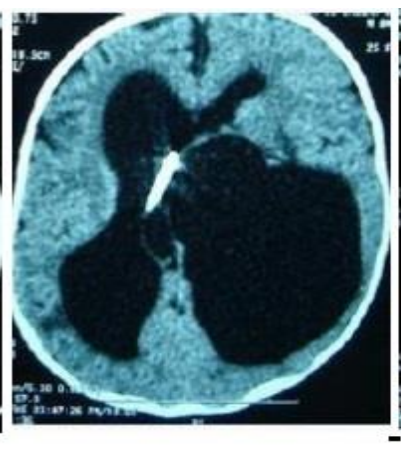

(c)

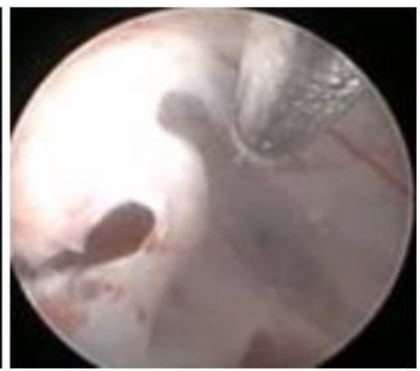

(g)

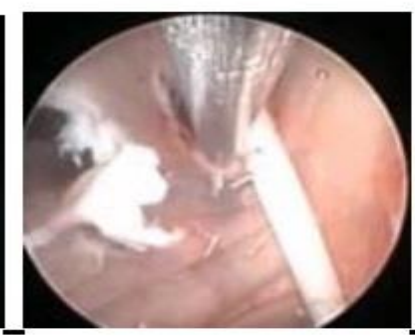

(k)

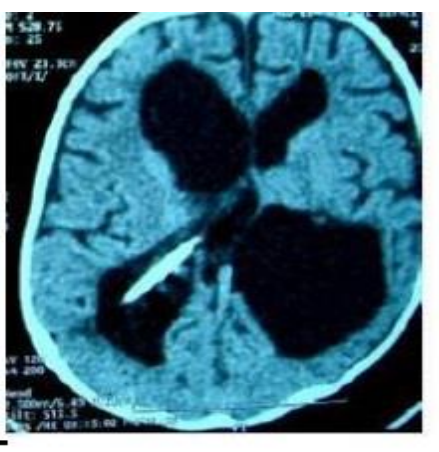

(d)

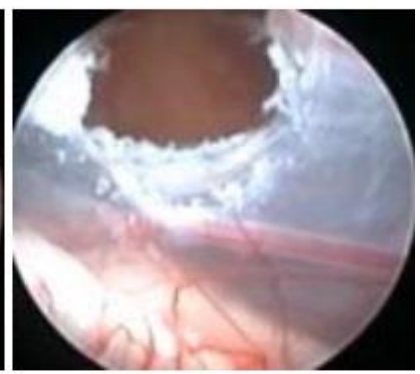

(h)

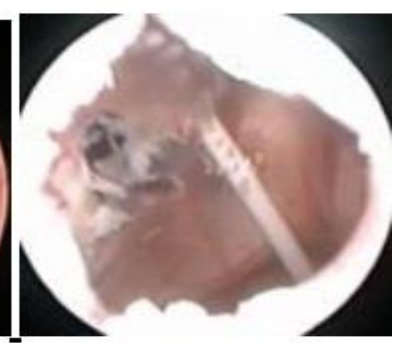

(I)

FIGURE 1. a-l: Radiological and intraoperative endoscopic photographs of a case of shunt related cyst. a) Preoperative CT. b) CT after insertion of a shunt. c) Three months follow up CT after shunt insertion with development of shunt related cyst. d) CT after 
endoscopic fenestration and shunt revision with improvement of hydrocephalus e) Endoscopic view of the cyst wall. f) Opening of the cyst. g) Coagulation and shrinkage of the wall of the cyst. h) Fenestrated cyst with adherent shunt. i) Shunt adherent to the inner cyst wall. j) Occlusion of the shunt perforations by proliferation of the cyst wall. k) Sharp dissection of the shunt from the adherent cyst. I) Endoscopic view after shunt revision and cyst perforation.

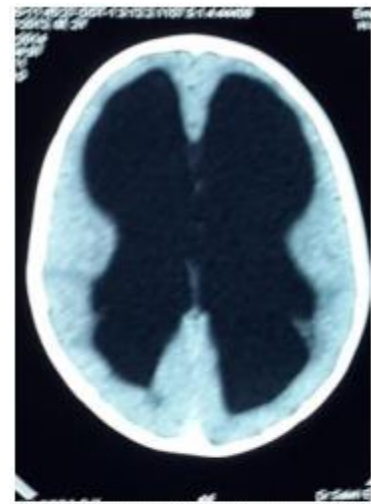

(a)

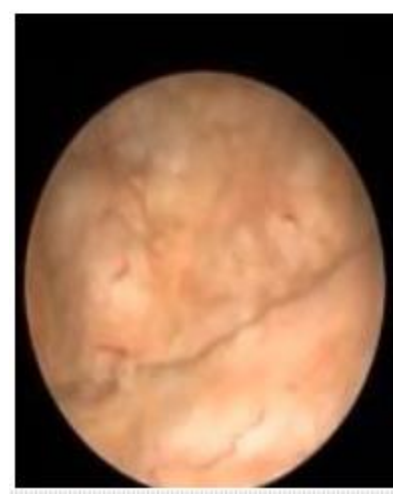

(e)

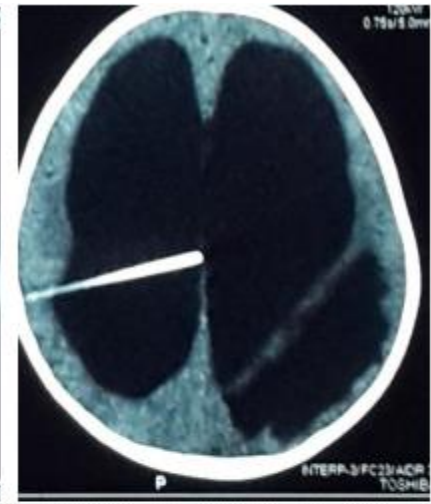

(b)

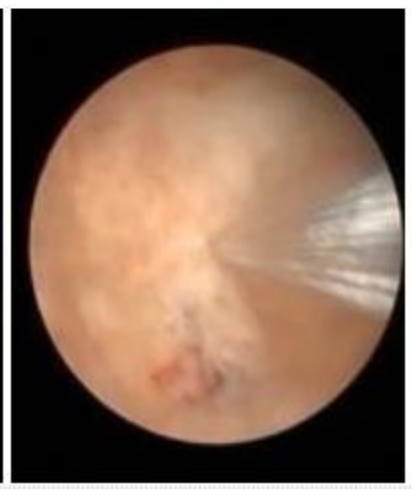

(f)

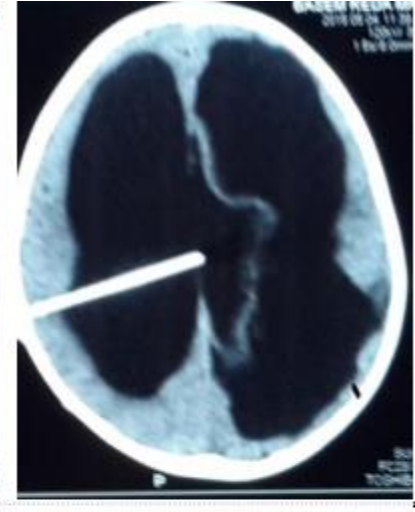

(c)

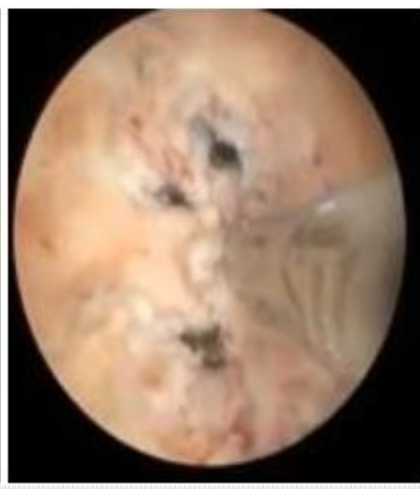

(g)

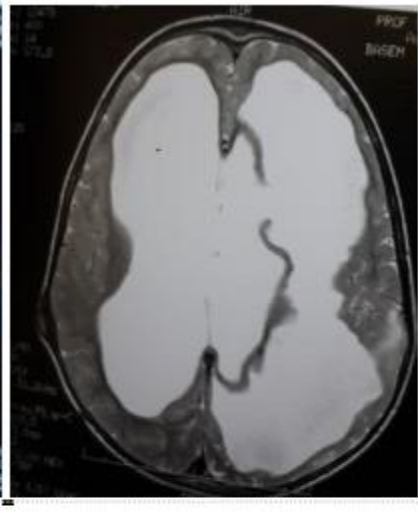

(d)

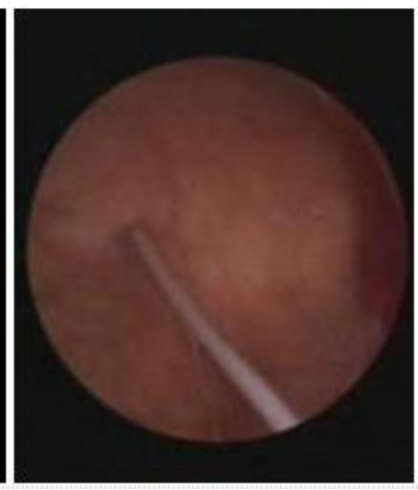

(h)

FIGURE 2. a-f: Radiological and endoscopic photographs of a case of post-haemorrhagic hydrocephalus. a) Preoperative CT. b) CT after insertion of the shunt with early formation of a cyst. c) CT after three months with progressive cyst enlargement. d) T2-MRI after cyst fenestration with good communication. e) Endoscopic view of thick cyst wall. f) Bipolar perforation of the cyst wall. g) Sharp dilatation of the stoma. h) Endoscopic view of the shunt within the ventricle through the performed stoma.

\section{DISCUSSION}

Uniloculated intraventricular discrete CSF filled compartment has been defined as a particular diagnostic entity by Cushing since he reported his first case of intraventricular unilocular compartment [1]. Subsequently Salmon in 1970 reported five cases of uniloculated hydrocephalus [17].

Non-colloidal neuroepithelial cysts are the most common underlying etiological causes for uniloculated hydrocephalus which may lead to obstruction of normal ventricular pathways with subsequent cystic dilation of one segment of the ventricular system, e.g. foramen of Monro or aqueduct and formation of uniloculated compartment. Excess CSF drainage via previously inserted ventricular catheters within a previously communicating ventricular system can cause ventricular morphological changes and possibly leads to isolation of ventricular compartments. Moreover, CSF over drainage might result in unilateral ventricular collapses or obstruction of foramen of Monro or aqueduct [4, 7].

Failure of the previous shunt may result from collapse of the isolated compartment around the tip of the catheter with subsequent obliteration of ventricular catheter fenestrations by reactive proliferation of fibrillary astrocytes [12].

Although the pathogenesis of univentricular 
loculation is not well known but the most accepted theory is that the inflammatory reaction related to previous shunt insertion, infection and intraventricular hemorrhage are the triggering factors for subendymal gliosis that may enhance the formation of glial tufts which occlude the holes of the ventricular system and act as a nidus for ventricular septations [11, 18]. Regardless of the accurate mechanism, the problem progress steadily toward greater complexity with time and traditional treatment ultimately require repeated surgeries with increasingly complex shunt devices [1].

The synonyms and classification of complex hydrocephalus are still interchangeable and unclear in the literatures. Spennato et al. published a classification for variable types of ventricular loculation defined by the location and number of compartments [19]. According to the previous proposed scheme "uniloculated" and "multiloculated" are broadly defined based on anatomical factors only [1]. These categories should be further subdivided into physiologically simple or physiologically complex based on whether CSF absorption is normal or impaired [2]. Differentiation between the previous entities is of great importence as their pathogenesis, surgical outcome and prognosis are quite different [6].

The clinical features of loculated hydrocephalus are not specific and are difficult to recognize because they often arise in a neurologically compromised infant or child with a previous history of hydrocephalus, neonatal meningitis or intraventricular haemorrhage. In our study the progressive head enlargement was the most common presenting symptom in 28 cases (63.6\%) followed by seizures in 14 cases (31.8\%) headache in 10 cases $(22.7 \%)$, focal neurological deficit in 10 cases (22.7\%) and gait disturbance in 6 cases (13.6\%).

Intraventricular loculation with previous ventriculoperitoneal shunt is a complex disorder that is difficult to treat. Those patients usually require repeated shunt revisions. Furthermore, those patients may need division and lysis of intraventricular septations either surgically through transcallosal craniotomy or through endoscopic techniques $[13,16]$.

The best advantages of the endoscopic procedures over transcallosal craniotomy are limited invasiveness and short postoperative recovery time. Nowadays the recent technical improvement in endoscopic techniques and high morbidity associated with multiple shunt revision or transcallosal craniotomy has shifted neurosurgeon toward the minimally invasive endoscopic techniques for lysis of intraventricular septae simultaneously with endoscopic shunt revisions [5].

Our objective in management of a single intraventricular loculation in a previously shunted patient was to restore the continuity between the isolated compartment and the ventricular system cavity to create a single cavity with the intent of simplifying or removal of the previous shunt system and reducing the need for repeated procedure. The proposed goal has been achieved in thirty-six patients (81.8\%) with simplification of the previous shunts in 28 patients (63.6\%); out of them eight patients (18.2\%) became shunt independent after endoscopic cyst fenestration and additional endoscopic foraminoplasty, septum pellucidotomy or third ventriculostomy.

Shunt malfunctioning was found in most cases of our series (34 cases, 77\%) due to either collapse and adhesive scarring of the cyst around the catheter tip or occlusion of the catheter perforations by debris or proliferating epithelial tufts. We used bipolar coagulation for safe dissection of the catheter tip from the adherent collapsed cyst wall to avoid catastrophic intraventricular bleeding which may happen in absence of endoscopic control.

In his series of 31 pediatric patients with uniloculated hydrocephalus, El-Ghandour treated 9 cases of uniloculated hydrocephalus with preexisting shunt system demonstrating reduction of shunt revision rate from 2.7 per year before fenestration to 0.25 per year after fenestration with simplification of four previous complex shunt systems and all patients required single repeated endoscopic procedures [6]. Lewis et al. reported 34 cases of both multiloculated and uniloculated hydrocephalus and shunt revision rate dropped from $3.04 \%$ prior to endoscopy to 0.25 after the procedure and $50 \%$ of the patients shunted prior to endoscopy required repeat endoscopic procedures [12].

In our series 90 revisions were required in 32 patients prior to endoscopy with shunt revision rate was 2.98/year which have been reduced to 0.27 after endoscopic fenestration as only four patients required endoscopic shunt revision. Ten patients (22.7\%) of our series required fourteen repeated endoscopic procedure for opening of a closed 
fenestrae or endoscopic shunt revision. The explanation of small percentage of repeated endoscopic procedure, in our cases compared by previously mentioned series is that we have 20 cases of neuroepithelial cysts presented to us with malfunctioning shunts and none of them required to repeat surgery. Moreover, we have only 12 cases of post meningitis and post haemorrhagic uniloculated hydrocephalus in which 8 cases required 12 repeated endoscopic surgery and only two patients with shunt related cysts had a single repeated endoscopic surgery

During repeated endoscopic procedures, ten cysts required reopening of closed stoma and four ventricular catheters required reopening of the closed stoma either due to gliosis in two cases or obstruction of the catheter openings by a debris in the other cases. Ultimately, eight patients (18.2\%) "Six with post-meningitis loculations and two with post-haemorrhagic loculations" required insertion of additional ventricular catheters after failure of repeated endoscopic procedures to achieve proper communication with persistent undrained intraventricular loculated compartments.

In the context with the experience of other endoscopic surgeons, we found that sharp dissection, employment of wide fenestrations of at least one $\mathrm{cm}$ and coagulation of the cysts wall ensured the success of the procedure and reduced the chance of recurrence $[10,15]$.

Previous series of endoscopic fenestration of loculated hydrocephalus highlighted the combination of endoscopic procedures to achieve better success and moreover may help shunt independence $[6,20]$.The obvious advantages of leaving one shunt in complex hydrocephalus are fewer opportunities for obstruction, rupture, infection and gliosis [3].

Septum pellucidotomy, foraminoplasty and third ventriculostomy were employed in our cases in combination with endoscopic cyst fenestration and shunt revision that have been succeed to achieve simplification of the previous shunt systems in $(63.6 \%)$ and elimination of shunt dependency in eight patients (18\%). The immaturity and deficient absorptive capabilities of scared subarachnoid space by a previous inflammatory process may be responsible for the lower shunt independent incidence in our study in line with others series $[2$, 12].
Nida and Haines who advocate transcallosal craniotomy for division and lysis of intraventricular septation criticized the endoscopic approaches by their limited ability to control intraoperative bleeding which may obscure the operative field [13]. However, intraoperative haemorrhage presented no great problem and did not result in termination of any procedure in this series. Haemorrhage was controlled by continuous irrigation with warm lactated ringer solution and occasionally monopolar and bipolar coagulation were helpful to control any bleeding point. Moreover, proper coagulation of site of fenestration may help to minimize the incidence of intraoperative bleeding.

As regard clinical outcome 28 patients (63.6\%) who presented with head enlargement had stabilization or decline in their head circumference. Epilepsy was controlled in ten patients (72\%) out of fourteen patients presented with seizures. Focal neurological deficient was improved in six patients (60\%) and gait disturbance was improved in four patients (67\%). Development delay was excluded from postoperative assessment as developmental improvement is not correlated with the change of the cyst size. Our results are going with the previous series which assessed the postoperative clinical outcome, in addition to radiological improvement of hydrocephalus [6].

Our morbid complications were minimal and reversible including CSF leakage in two cases which stopped within few days after medical therapy, four cases of subdural hygroma that resolved spontaneously during follow up and only two cases of superficial wound infection which improved with antibiotic and repeated dressing.

\section{CONCLUSION}

Intraventricular loculations in previously shunted patients is among the most challenging scenarios encountered by a neurosurgeon. This complex disorder has a relatively evolving course in the form of ongoing worsening with time that requires increasingly difficult surgical procedures. The main objective in management of such cases is to reduce the number of surgical procedures more than improving the quality of life of the patient. Endoscopic surgical techniques are attractive minimal invasive alternatives that may help to achieve treatment objectives with minimal postoperative morbidity. 


\section{AUTHORS CONTRIBUTIONS}

This work was carried out in collaboration between all authors. Author Amr Farid, designed the study, Author Mohamed State wrote the protocol, Author Ahmed Zaher managed the literature research, Author Hatem Badr revised the final manuscript. All surgical procedures were carried out by single surgeon and assisted by the same surgical team including the four authors. All authors read and approved the final manuscript.

\section{REFERENCES}

1. Akbari S H A, Holekamp T F, Murphy T M, Mercer D, Leonard J R, Smyth M D, Park T, and Limbrick D D: Surgical management of complex multiloculated hydrocephalus in infants and children. Child's Nervous System 2015, 31(2), 243-249.

2. Andresen $M$ and Juhler M: Multiloculated hydrocephalus: $a$ review of current problems in classification and treatment. Child's Nervous System 2012, 28(3), 357-362.

3. Cipri S, Gambardella G, and Gangemi M: Neuroendoscopic approach to complex hydrocephalus: Personal experience and preliminary report. Journal of neurosurgical sciences 2001, 45(2), 92.

4. Cushing H: Surgery of the head. Surgery: its principles and practice 1908, 3, 255-256.

5. El-Ghandour $\mathrm{N} \mathrm{M}$ : Endoscopic cyst fenestration in the treatment of multiloculated hydrocephalus in children. Journal of Neurosurgery: Pediatrics 2008, 1(3), 217-222.

6. El-Ghandour N M: Endoscopic cyst fenestration in the treatment of uniloculated hydrocephalus in children. Journal of Neurosurgery: Pediatrics 2013, 11(4), 402-409.

7. Eller $\mathrm{T} W$ and Pasternak J F: Isolated ventricles following intraventricular hemorrhage. Journal of neurosurgery 1985, 62(3), 357-362.

8. Gangemi M, Maiuri F, Donati $P$, and Sigona L: Neuroendoscopy: Personal experience, indications and limits. Journal of neurosurgical sciences 1998, 42(1), 1.

9. Greenfield J P and Souweidane $M$ M: Endoscopic management of intracranial cysts. Neurosurgical focus 2005, 19(6), 1-9.
10. Heilman C B and Cohen A R: Endoscopic ventricular fenestration using a "saline torch". Journal of neurosurgery 1991, 74(2), 224-229.

11. Jamjoom A, Mohammed A, Al-Boukai A, Jamjoom Z, Rahman $\mathrm{N}$, and Jamjoom $\mathrm{H}$ : Multiloculated hydrocephalus related to cerebrospinal fluid shunt infection. Acta neurochirurgica 1996, 138(6), 714-719.

12. Lewis A I, Keiper G L, and Crone K R: Endoscopic treatment of loculated hydrocephalus. Journal of neurosurgery 1995 , 82(5), 780-785.

13. Nida $T Y$ and Haines $S \mathrm{~J}$ : Multiloculated hydrocephalus: craniotomy and fenestration of intraventricular septations. Journal of neurosurgery 1993, 78(1), 70-76.

14. Oi S and Abbott R: Loculated ventricles and isolated compartments in hydrocephalus: their pathophysiology and the efficacy of neuroendoscopic surgery. Neurosurgery clinics of North America 2004, 15(1), 77-87.

15. Oi S, Hidaka M, Honda Y, Togo K, Shinoda M, Shimoda M, Tsugane R, and Sato O: Neuroendoscopic surgery for specific forms of hydrocephalus. Child's Nervous System 1999, 15(1), 56-68.

16. Powers S; Fenestration of intraventricular cysts using a flexible, steerable endoscope, in Minimally Invasive Neurosurgery I. 1992, Springer. p. 42-46.

17. Salmon J H: Isolated unilateral hydrocephalus following ventriculoatrial shunt. Journal of neurosurgery 1970, 32(2), 219-226.

18. Schulz M, Bohner G, Knaus $H$, Haberl $H$, and Thomale U-W: Navigated endoscopic surgery for multiloculated hydrocephalus in children. Journal of Neurosurgery: Pediatrics 2010, 5(5), 434-442.

19. Spennato P, Cinalli G, Ruggiero C, Aliberti F, Trischitta V, Cianciulli $E$, and Maggi G: Neuroendoscopic treatment of multiloculated hydrocephalus in children. Journal of Neurosurgery: Pediatrics 2007, 106(1), 29-35.

20. Teo C, Kadrian D, and Hayhurst C: Endoscopic management of complex hydrocephalus. World neurosurgery 2013, 79(2), S21. e1-S21. e7. 